\title{
Potential Application of a Visible Light-Induced Photocured Hydrogel Film as a Wound Dressing Material
}

\author{
Aazadehsadat Hashemi Doulabi, ${ }^{1}$ Hamid Mirzadeh, ${ }^{1}$ Nasrin Samadi, ${ }^{2}$ \\ Shadab Bagheri-Khoulenjani, ${ }^{1}$ Mohammad Atai, ${ }^{3}$ and Mohammad Imani ${ }^{4}$ \\ ${ }^{1}$ Department of Polymer and Color Engineering, Amirkabir University of Technology, P.O. Box 15875-4413, Tehran 15916-34311, Iran \\ ${ }^{2}$ Department of Drug and Food Control, Faculty of Pharmacy and Biotechnology Research Center, \\ Tehran University of Medical Sciences, P.O. Box 14155-6451, Tehran 14176-14411, Iran \\ ${ }^{3}$ Department of Science, Iran Polymer and Petrochemical Institute, P.O. Box 14965-115, Tehran 14977-13115, Iran \\ ${ }^{4}$ Department of Novel Drug Delivery Systems, Iran Polymer and Petrochemical Institute, P.O. Box 14965-115, Tehran 14977-13115, Iran
}

Correspondence should be addressed to Mohammad Imani; m.imani@ippi.ac.ir

Received 29 May 2015; Revised 9 August 2015; Accepted 11 August 2015

Academic Editor: Iliya Rashkov

Copyright (C) 2015 Aazadehsadat Hashemi Doulabi et al. This is an open access article distributed under the Creative Commons Attribution License, which permits unrestricted use, distribution, and reproduction in any medium, provided the original work is properly cited.

\begin{abstract}
The objective of this work was to prepare hydrogel films, as semi-interpenetrating polymer networks (semi-IPN), based on polyethylene glycol-co-fumarate (PEGF) and chitosan $(\mathrm{Ch})$ blends. Hydrogel films were prepared by free radical cross-linking of PEGF, an unsaturated aliphatic polyester, in the presence of $N$-vinyl pyrrolidone (NVP), camphorquinone (CQ), and $N, N$-dimethylp-toluidine (DMPT) as a cross-linking, photoinitiating, and accelerating agent, respectively. The effect of NVP concentration on physicochemical and biological properties of semi-IPN film properties was evaluated. The sol fraction, water vapor transmission rate, and swelling degree of the hydrogel films were also investigated. Antibacterial activity against $S$. aureus was observed for the photocured blend hydrogels of Ch/PEGF with no toxicity to L929 cells according to the cell viability assays. Blend hydrogel films showing $600 \pm 88 \%$ of equilibrium swelling degree in water and the lowest sol fraction $(3.14 \pm 1.22 \%)$ were obtained at $20 \mathrm{wt} \%$ of NVP content whilst preserving their own cytocompatibility and antibacterial activity. Therefore, this formulation was considered as an optimal semi-IPN blend hydrogel film composition with potential application for wound dressing.
\end{abstract}

\section{Introduction}

There is an increasing interest in preparing of polymer blends based on synthetic resins and natural biopolymers for biomedical applications such as wound dressing $[1,2]$. Recently, a lot of literature surveys covering traditional treatments to modern and sophisticated techniques including several natural and synthetic polymers have been published in wound dressing fields [3]. The preliminary requirements for a wound dressing composition intended for soft tissue applications are as follows: biocompatibility, nontoxicity, appropriate gas permeability, easy application and removal without causing secondary trauma to the wound, and providing suitable microenvironment for wound healing just to name a few $[1,4]$. Using polymer blends seems advantageous in this regard and could secure more aforementioned requirements due to the improved properties not attainable by a single polymer.

Chitosan $(\mathrm{Ch})$, the linear and partly deacetylated derivative of chitin, has recently received great attention for different biomedical applications due to its irreplaceable and intrinsic properties [5]. It exhibits some properties such as biodegradability, biocompatibility, nonantigenicity, nontoxicity, and intrinsic antibacterial activity; hence, this polysaccharide possesses a great potential to be used as a biomaterial for wound dressing applications [1]. Wound dressing with intrinsic antibacterial properties is preferable comparing to those loaded with antibacterial agents especially from safety viewpoint $[1,4]$. Ch, however, requires modification due to its inferior mechanical properties. 
Several approaches including Ch blending with other synthetic or natural polymers have been examined to obviate these drawbacks while improving other properties. It can provide the wide range of physicochemical properties, processing techniques of synthetic polymers, and the biocompatibility and biological interactions of natural polymers simultaneously [6-9]. Blending $\mathrm{Ch}$ with synthetic polymers such as poly(ethylene oxide), poly(vinyl alcohol), and polylactic acid gives just a few examples of these approaches [10-15]. Our group has reported the preparation of a novel blend film of $\mathrm{Ch} /$ poly(ethylene glycol fumarate) (Ch/PEGF) and evaluated its properties as a dressing $[1,16]$. PEGF is an unsaturated polyester showing good biocompatibility, cytocompatibility, and biodegradability profile whilst being able to be photocured even with visible light irradiation $[17,18]$. It also helps $\mathrm{Ch}$ to become ductile, in a blend, due to the presence of flexible PEG chains in its backbone [19]. Moreover, it causes enhancing moisture penetration and consequently increasing water vapor transmission rate (WVTR) value due to the existence or progress in intermolecular interactions between Ch and PEGF molecules $[1,16]$.

PEGF, as a water soluble polymer, would be removed from its $\mathrm{Ch}$ matrix even in the presence of hydrogen bonds with Ch chains, which affects blend properties to some extent [1, 20]. PEGF can be cross-linked via free radical mechanisms initiated by heat, redox reaction, and photoirradiation due to the unsaturated double bonds present in its backbone $[18,21]$.

The aim of this work was to prepare photocured Ch/PEGF blend films and evaluate their properties in biomedical applications as wound dressing films. To this end, semiinterpenetrating network (semi-IPN) hydrogels were prepared via photocuring of PEGF present in the Ch matrix to provide a readily adjustable network considering properties such as sol fraction, swelling degree, WVTR, antibacterial activity, and biological properties. Effects of photocuring agent concentration on physicochemical and biological properties of the samples were evaluated and one specific formulation was introduced as the optimum sample for wound dressing applications. To the best of our knowledge, photocurable blend films of Ch/PEGF and their wound healing performance have not been discussed elsewhere.

\section{Experimental}

2.1. Materials. Low viscosity $\mathrm{Ch}(20-200 \mathrm{mPa} \cdot \mathrm{s}, \mathrm{DDA}=80 \%$, Fluka, Germany) was purified as reported elsewhere [22]. PEGF $\left(M_{w}: 19 ; M_{n}: 16 \mathrm{kDa}\right.$; degree of oligomerization: $\left.\approx 4\right)$ was synthesized in house by condensation polymerization of PEG diol $\left(M_{w}=3 \mathrm{kDa}\right.$, Ph Eur grade, Merck Chemicals, Dusseldorf, Germany) and fumaryl chloride $95 \%$ in the presence of propylene oxide 99\% (Aldrich, Milwaukee, MN, USA) as catalysts and proton scavenger according to the procedure described in detail elsewhere [18]. Camphorquinone $97 \%$ (CQ) was purchased from Aldrich, USA. Sodium hydroxide, $N$-vinyl pyrrolidone (NVP), N,N-dimethyl-p-toluidine $99 \%$ (DMPT), methylene chloride, and acetic acid were obtained from Merck Chemicals, Germany. Fumaryl chloride and NVP were purified by distillation as mentioned in detail, previously [18]. All of the other chemicals and reagents were of analytical grade and used as received without further purification.

\subsection{Methods}

2.2.1. Semi-IPN Ch/PEGF Film Preparation. According to our previous studies, 80 to $20 \mathrm{Ch} / \mathrm{PEGF}$ blend ratio (w/w) was selected for preparing of samples reported here $[1,16,22]$. $\mathrm{Ch} / \mathrm{PEGF}$ blend solution was prepared by dissolving $\mathrm{Ch}$ and PEGF in $1 \%(\mathrm{v} / \mathrm{v})$ acetic acid and then let it reach concentrated form under stirring. To this end, CQ (1 wt \% relative to PEGF), NVP $(10,20$, and $40 \mathrm{wt} \%$ relative to PEGF), and DMPT $(1 \mu \mathrm{L})$ were mixed and then added to the Ch/PEGF viscous solution. The blend was photocured similar to the procedure reported with some modifications [18]. The solutions were cast in leveled, polystyrene dishes and cured for $200 \mathrm{~min}$ in a photocuring box (TRAY-LUX, M5+, Taiwan). After completion of the curing reaction, the films were dried at room temperature for $48 \mathrm{~h}$ and then kept in a refrigerated desiccator at $5^{\circ} \mathrm{C}$ until being used.

2.2.2. Gel Permeation Chromatography. Gel permeation chromatography (GPC) was performed using a GPC instrument (Shimadzu, CR4AX, GC15A, Japan). After obtaining the calibration curve with polystyrene standards with known molecular weights, $M_{n}$ and $M_{w}$ were calculated by running PEGF sample. THF was used as the mobile phase eluting at a flow rate of $1.0 \mathrm{~mL} \mathrm{~min}^{-1}$. A $100 \mu \mathrm{L}$ sample of $0.1 \mathrm{mg} \mathrm{mL}^{-1}$ solution of the macromer in THF, which was filtered through a $0.22 \mathrm{~mm}$ filter prior to use, was injected for the measurement.

2.2.3. Degree of Conversion. Degree of conversion (DC\%) of the semi-IPN networks was determined by FTIR spectroscopy. FTIR spectra $\left(4000-400 \mathrm{~cm}^{-1}\right)$ were acquired on a Bruker (Germany), Equinox 55 spectrophotometer at $4 \mathrm{~cm}^{-1}$ resolution and 32 scans. The specimens were examined as films at ambient temperature and their spectra were recorded before and after curing reaction. DC\% was determined from the ratio of absorbance intensities of aliphatic $\mathrm{C}=\mathrm{C}$ (peak positioned at $1645 \mathrm{~cm}^{-1}$ ) against an internal reference signal belonging to $\mathrm{CH}_{3} \mathrm{COH}$ group appearing at $851 \mathrm{~cm}^{-1}$ before and after curing of the specimen. The DC\% was then calculated using (1) [23]. Each FTIR spectrum was deconvoluted using Origin software to calculate $A_{1645}$ due to overlapping peaks:

$$
\begin{aligned}
& \text { DC } \%=1 \\
& -\left(\frac{\left(1645 \mathrm{~cm}^{-1} / 851 \mathrm{~cm}^{-1}\right) \text { peak area after curing }}{\left(1645 \mathrm{~cm}^{-1} / 851 \mathrm{~cm}^{-1}\right) \text { peak area before curing }}\right) \\
& \times 100 .
\end{aligned}
$$

2.2.4. Sol Fraction. To investigate the leachable residues in the blend, sol fraction (SF) was determined as follows. First, the residual solvent was removed by immersing the films in 
methanol, as a nonsolvent, for $24 \mathrm{~h}$. Then, the samples were taken out and dried in a vacuum oven $\left(T=35^{\circ} \mathrm{C}, P=0.2 \mathrm{bar}\right)$ overnight and weighed $\left(W_{i}\right)$. Subsequently, the films were immersed in distilled water for $24 \mathrm{~h}$, dried in vacuo overnight, and then weighed again $\left(W_{d}\right)$. The SF was calculated using [10]

$$
\operatorname{SF}(\%)=\frac{\left(W_{i}-W_{d}\right)}{W_{i}} \times 100 .
$$

2.2.5. Equilibrium Swelling Degree. Equilibrium swelling degree (ESD) was determined using a gravimetric method. The specimens were cut into $1 \mathrm{~cm} \times 1 \mathrm{~cm}$ rectangles and then dried in vacuo $\left(T=35^{\circ} \mathrm{C}, P=0.2 \mathrm{bar}\right)$ for $24 \mathrm{~h}$ and weighted $\left(W_{0}\right)$. Afterward, the specimens were immersed in an excess amount of PBS $(0.1 \mathrm{M}, \mathrm{pH}=7.4)$ at $25^{\circ} \mathrm{C}$ until an equilibrium was reached ( $c a .24 \mathrm{~h}$ ) when surface of the samples was blotted by a filter paper and weighted $\left(W_{E}\right)$ again. The ESD was calculated using [4]

$$
\operatorname{ESD}(\%)=\frac{\left(W_{E}-W_{0}\right)}{W_{0}} \times 100 .
$$

2.2.6. Water Vapor Transmission Rate. To determine gas permeability of the blend films, WVTR of the sample films was determined according to ASTM E96/E96M-10 [24] procedure with minor modifications described elsewhere [1]. Briefly, a glass bottle containing anhydrous silica gel desiccant was covered by the films under test $(15 \mathrm{~mm}$ diameter of exchange area) and sealed using paraffin wax. The assembly was weighed 8 times at $1 \mathrm{~h}$ intervals and kept in a humidity chamber maintaining $75 \pm 3 \%$ of relative humidity ( $T=25 \pm$ $\left.1^{\circ} \mathrm{C}\right)$ using standard saturated solution of sodium chloride. Weight variations of the bottles were plotted versus time. The WVTR was determined as [24]

$$
\text { WVTR }=\frac{\text { slop } \times 24}{S}\left[\mathrm{~g} / \mathrm{m}^{2} \text { day }\right],
$$

where $S$ is the vapor exchange area under test. Control cups, without the anhydrous silica gel desiccant, were conducted in parallel. The WVTR experiment was repeated thrice for each formulation.

2.2.7. Antibacterial Activity. Antibacterial activity was determined by tracing the viable cell count after exposure of test specimens $(\Phi=25 \mathrm{~mm})$ with Staphylococcus aureus (ATCC 6538) as a Gram-positive bacterium. An overnight culture of $S$. aureus bacterium was used to prepare a bacterial suspension with a cell density approximately equivalent to $10^{8} \mathrm{CFU} \mathrm{mL}{ }^{-1}$. The test samples were decontaminated by immersing in $70 \%(\mathrm{v} / \mathrm{v})$ ethanol for $10 \mathrm{~min}$ and then washing with sterile phosphate buffer solution and air-drying under laminar air flow [25]. Each film was added to $10 \mathrm{~mL}$ of nutrient broth medium (Merck, Germany) which was then inoculated with $S$. aureus suspension to reach the final concentration of $10^{7} \mathrm{CFU} \mathrm{mL} \mathrm{m}^{-1}$. After $24 \mathrm{~h}$ exposure at room temperature, each sample was serially diluted and the number of survived bacteria was determined by pour plate method using nutrient agar medium (Merck, Germany). Plates were incubated at $37^{\circ} \mathrm{C}$ for $48 \mathrm{~h}$ and the total viable bacterial counts were determined and expressed as $\mathrm{CFU} \mathrm{mL} \mathrm{m}^{-1}$ [26]. Nutrient broth containing the same bacterial inoculum and no specimen was considered as control. Equations (5) and (6) were used to calculate the killing efficacy (Kill\%) and the population reduction of the test organism, respectively [27],

$$
\begin{aligned}
& \text { Kill\% } \\
& =\frac{\text { cell count of control }- \text { cell count of sample }}{\text { cell count of control }} \\
& \quad \times 100,
\end{aligned}
$$

Log population reduction

$=$ Log cell count of control

- Log survivor count on sample.

2.2.8. Cell Culture. Mouse fibroblast cells (L929) were generously provided by National Institute of Genetic Engineering and Biotechnology. The cells were cultured in a medium consisting of Roswell Park Memorial Institute (RPMI-1640, Sigma Chemical Company, St. Louis, USA) supplemented with $10 \%$ heat-inactivated fetal bovine serum (Gibco, BRL) and streptomycin $100 \mu \mathrm{g} \mathrm{mL}^{-1}$ and penicillin $100 \mathrm{IU} \mathrm{mL}^{-1}$ (Sigma Chemical Company, St. Louis, USA). A routine subculture was used to maintain the cell line [28]. The cells were incubated in a humidified atmosphere of $5 \% \mathrm{CO}_{2}$ at $37^{\circ} \mathrm{C}$. After one week of incubation, the cell monolayer was harvested by trypsinization. Ch/PEGF semi-IPN films were cut in circular pieces $(\Phi=0.8 \mathrm{~cm})$ and sterilized by immersing in $70 \%(\mathrm{v} / \mathrm{v})$ ethanol and sterile phosphate buffer saline consecutively for ten minutes. The samples were placed separately into a multiwell plate with $5 \mathrm{~mL}$ of cell suspension and seeded, keeping one well as control without any sample, and then maintained in an incubator for $24 \mathrm{~h}$. The samples were examined by a light microscope (Nikon, E200, Japan) equipped with CCD camera (Micros, Smart eye, Austria).

To examine cytotoxicity (i.e., mitochondrial function), reduction of the MTT reagent (3-[4,5-dimethylthiazol-2yl]-2,5-diphenyltetrazolium bromide, Merck, Germany) was assessed as an assay of mitochondrial redox activity [29]. For the assay, a cell suspension was prepared and seeded onto 96-well plates and then incubated. After $24 \mathrm{~h}$ of culture, the supernatant of cell culture was removed and $100 \mu \mathrm{L}$ of MTT solution ( $5 \mathrm{mg} \mathrm{mL}^{-1}$ phosphate buffer saline) was added to each sample and incubated for $4 \mathrm{~h}$ at $37^{\circ} \mathrm{C}$, under a $\mathrm{CO}_{2}$ (5\%) atmosphere to dissolve the produced purple formazan crystals. Medium was aspirated and cultured fibroblasts were treated with $200 \mu \mathrm{L}$ of isopropanol/ $\mathrm{HCl}(0.04 \mathrm{~N})$ and incubated for $15 \mathrm{~min}$ at $37^{\circ} \mathrm{C}$. Then, $100 \mu \mathrm{m}$ of each well transferred to another 96-well plate. The optical density of the formazan solution was read on an ELISA reader (Microplate reader ELX808 Bio-Tek Instruments, USA) at $570 \mathrm{~nm}$ and the 


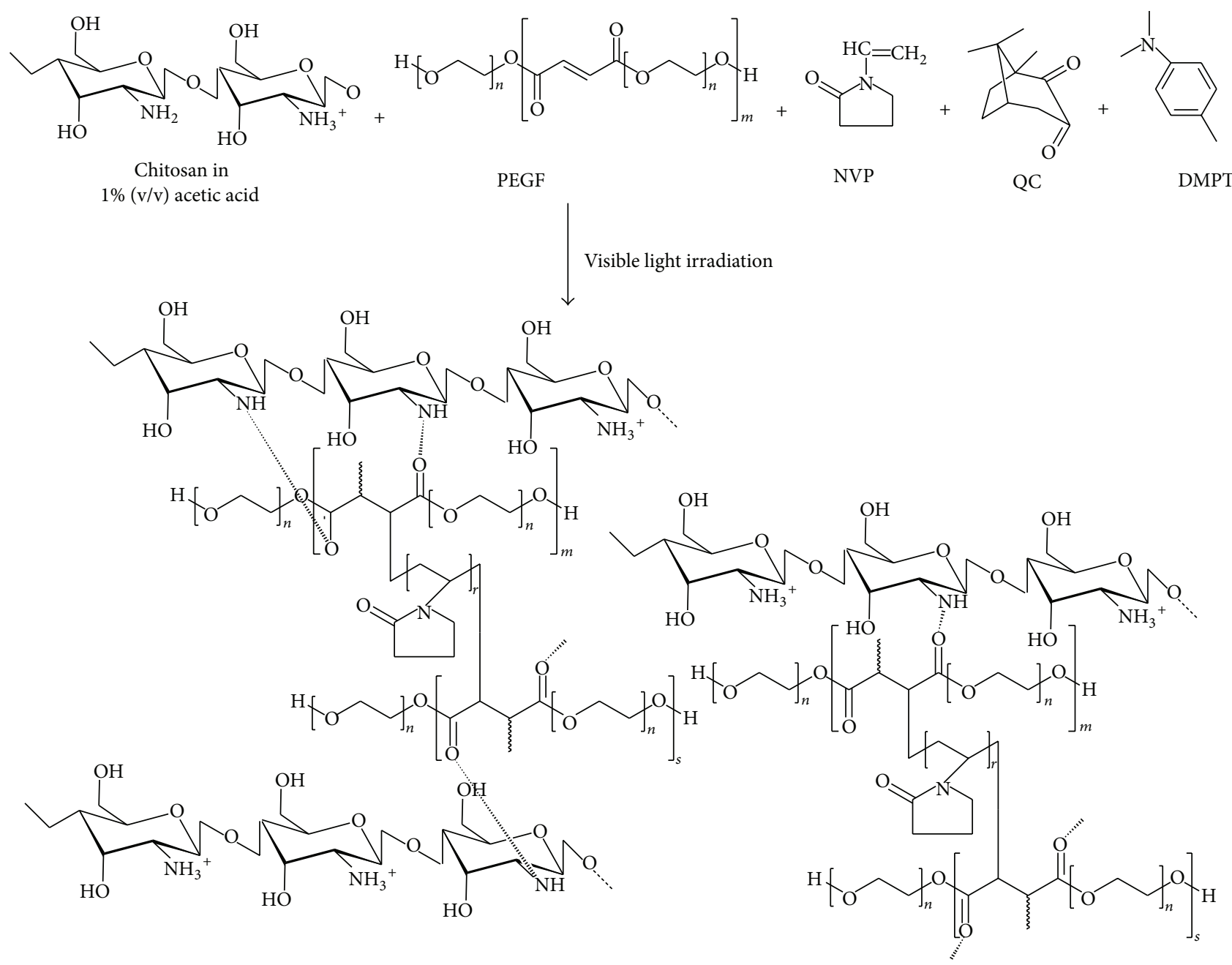

Photocured Ch/PEGF blend

SCHEME 1: Schematic representations of chemical structure of photocured blends of Ch/PEGF.

reference wavelength of $630 \mathrm{~nm}$. Controls without samples were carried out. The results were expressed as a percentage of cell viability.

2.3. Statistical Analyses. All experiments were performed on enough number of samples, that is, at least 3 samples. Differences between groups were analyzed by one-way Analysis of Variance (ANOVA). Linear regression was also used for data analysis. The Tukey ( $\alpha=0.05,95 \%$ confidence intervals) was also used to determine the significance of differences between specific means (Origin, 7.0, 2002, USA). All data were represented as mean $\pm \mathrm{SD}$.

\section{Results and Discussion}

This study was focused on the preparation and characterization of photocured film made of Ch/PEGF blend. Crosslinking of PEGF in the Ch matrix resulted in semi-IPN hydrogels (Scheme 1). Since CQ has an absorption peak at $467 \mathrm{~nm}$, this makes it more favorable for the lamps with emission peaks in the range of 470 to $500 \mathrm{~nm}$ [30]. Visible light (in blue region) was used for film curing which obviated the drawbacks of UV light to the surrounding tissues. In this paper, more important properties such as sol fraction, swelling degree, WVTR, antibacterial activity, biocompatibility, and cytocompatibility required for wound dressing applications have been evaluated.

3.1. Degree of Conversion. To determine light curing efficacy, double bond conversion was calculated using (1). The effect of NVP as a reactive diluent and cross-linking agent on the DC\% was depicted in Figure 1. With increasing in NVP concentration in feed from 10 to $20 \mathrm{wt} \%, \mathrm{DC} \%$ increased while by adding more NVP (up to $40 \mathrm{wt} \%$ ) it was decreased. The viscosity of the reaction medium plays an important role in the polymerization behavior [23]. The restricted mobility of the mixture at low NVP concentrations could affect the polymerization propagation and decrease the final conversion. Increasing in NVP content made the compound 


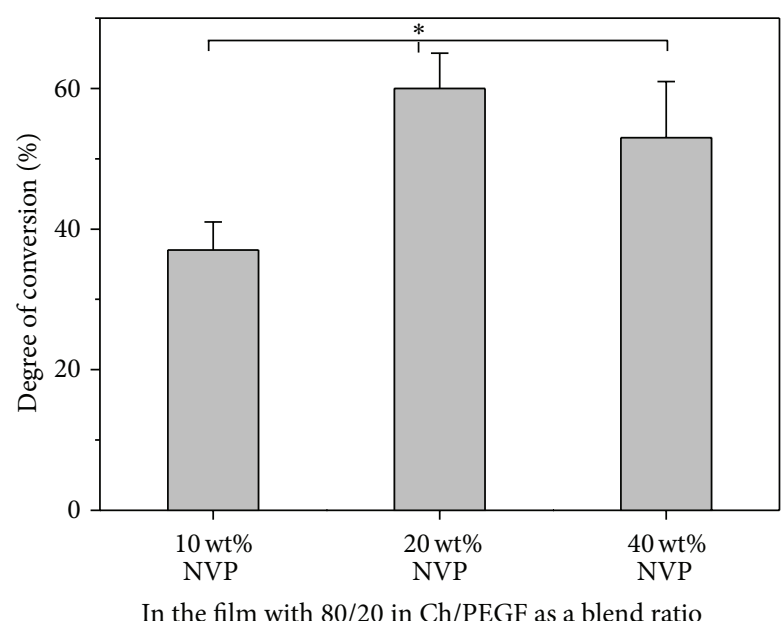

FIgURE 1: Degree of conversion of the blend films of Ch/PEGF $(80 / 20)$ with different contents of NVP as a cross-linking agent $\left({ }^{*} p<\right.$ $0.05)$.

(composed of PEGF-Ch-NVP-QC-DMPT-solution) low viscous; therefore, cross-linking agent was able to form more network bridges because of higher molecular mobility and finally DC\% increased. At higher concentrations of NVP (40 wt\%), a reduction in DC\% was observed which was due to formation of NVP homopolymers. The result is confirmed with considering the PEGF degree of oligomerization (or $\mathrm{C}=\mathrm{C}$ fumarate bond quantities) which will be mentioned in the next section. NVP is a monofunctional monomer with low polymerization rate and at higher concentrations the monomer tends to form more linear homopolymer chains rather than cross-linked network resulting in a decrease in final conversion [31]. The formation of the linear water soluble poly-NVP at high NVP concentrations was confirmed by sol fraction test.

3.2. Sol Fraction. Sol fraction of the uncured blend and photocured blends with different NVP contents (10, 20, and $40 \mathrm{wt} \%$ ) is determined as $11.6,7.2,3.1$, and $6.3 \%$ respectively, as shown in Figure 2. Photocuring of Ch/PEGF blends caused a decrease in the sol fraction of the blends due to formation of PEGF networks within Ch matrix. When semi-IPN Ch/PEGF was formed, free PEGF content decreased in comparison with uncured film; therefore, sol fraction decreased. By means of GPC $\left(M_{w}: 19, M_{n}: 16 \mathrm{kDa}\right)$ and then calculating degree of oligomerization $(\mathrm{C}=\mathrm{C}$ bonds quantities of PEGF), NVP $20 \mathrm{wt} \%$ was chosen as an optimum amount of cross-linking agent needed for photocuring. For cross-linking PEGF, the stoichiometric amount of NVP needed is equal to optimum $\mathrm{C}=\mathrm{C}$ bonds quantities of PEGF. Degree of oligomerization equals $M_{n \text { PEGF }} / M_{n \text { PEGFU }}$, where $M_{n \text { PEGF }}$ and $M_{n \text { PEGFU }}$ represent the number average molecular weights of PEGF and monomeric PEG fumarate unit, respectively [32]. GPC result showed that each PEGF chain has approximately four fumarate double bonds; therefore, the optimum required NVP for each mole PEGF was four moles. NVP with $20 \mathrm{wt} \%$ concentration could meet this demand. There have still been

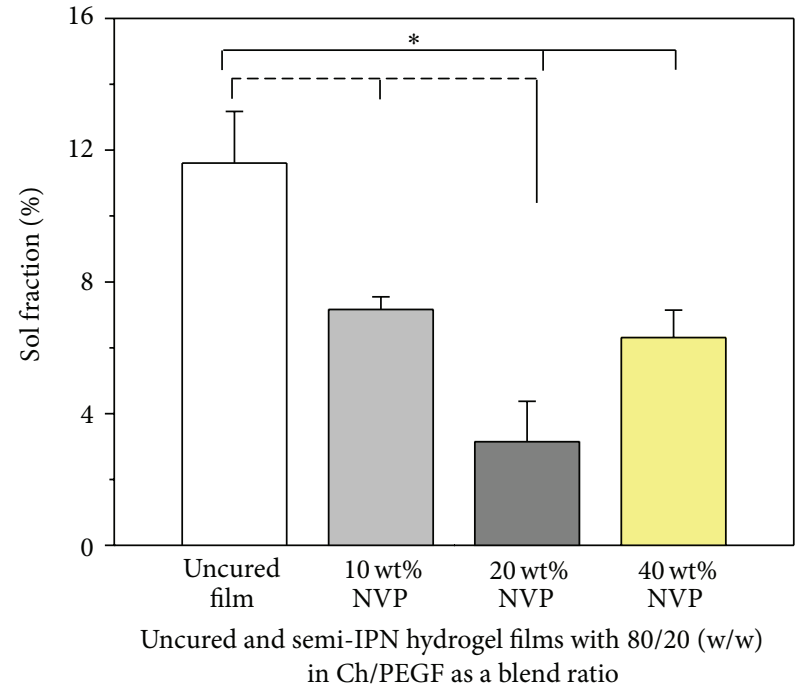

FIgURE 2: Sol fraction of photocured blend films of Ch/PEGF with $80 / 20$ in blend ratio containing different contents of NVP and uncured Ch/PEGF with $80 / 20\left({ }^{*} p<0.05\right)$.

some uncross-linked chains which dissolved in water when NVP was added in less than required amount (SF: 7.2\%). In line with this, adding NVP in more than double bond quantity, extra NVP formed water soluble homopolymer, that is, PVP [31] (SF: 6.3\%), as mentioned above. Hence, sol fraction of cured blends with 10 and $40 \mathrm{wt} \% \mathrm{NVP}$ is more than that of $20 \mathrm{wt} \%$ NVP (SF: 3.1\%) while all formulations of photocured blends show the SF less than that of uncured film $(p<0.05)$ as shown in Figure 2, indicating the photocured films have advantages on the uncured one.

3.3. Swelling Behavior. There are several types of wounds with different amount of exudates; therefore, exudate absorbing efficacy plays an important role for choosing an appropriate dressing to provide a proper moist environment to prevent the wound bed from dryness and also infection [1]. The swelling behavior of the films was investigated and reported as ESD. The water swelling of the semi-IPN is higher than the uncured films as shown in Figure 3. Since adequate exudate absorption is one of the essential properties for wound dressing, therefore there is another strong point of cured films in comparison with uncured ones. In the uncured film, after $24 \mathrm{~h}$ immersion in water to reach equilibration, the uncrosslinked PEGF chains were removed from the blend and left water insoluble $\mathrm{Ch}$ matrix. In the cured films, however, water molecules were diffused into and placed in the networks. With the increase of NVP concentration from 10 to $40 \mathrm{wt} \%$, swelling degree decreased due to higher cross-linking density of the network. This is in agreement with GPC result for degree of oligomerization. As mentioned before, the required NVP for each mole of PEGF was four moles (NVP with $20 \mathrm{wt} \%$ concentration). The values less than $20 \mathrm{wt} \%$ caused some uncross-linked PEGF remaining in the matrix which resulted in loose network and more swelling as shown in Figure 3 (ESD: 700\%). Sol fraction findings also confirmed 


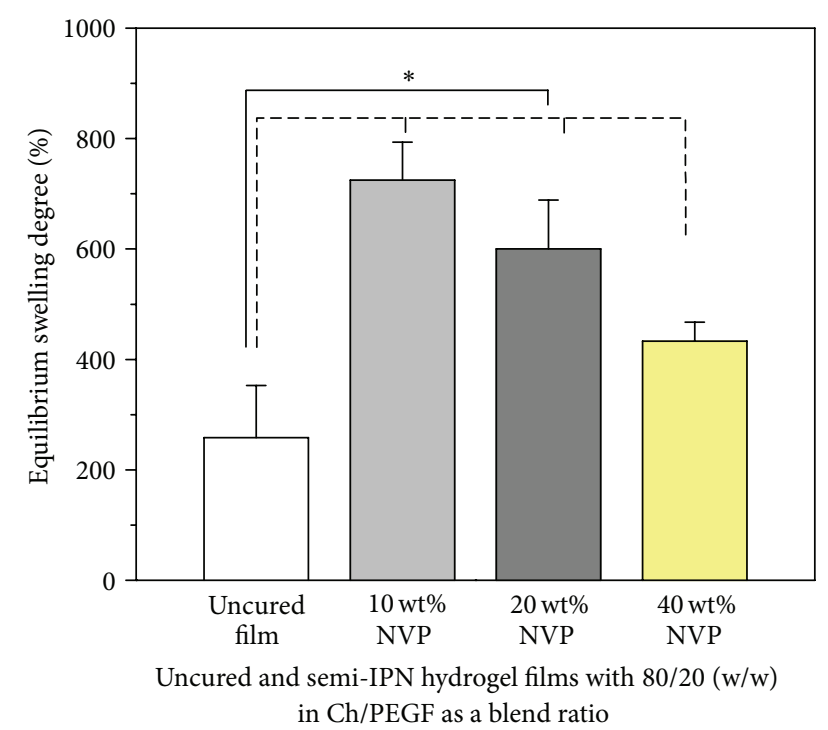

Figure 3: Swelling degree of photocured blend films of Ch/PEGF with $80 / 20$ in blend ratio containing different contents of NVP and uncured Ch/PEGF with $80 / 20\left({ }^{*} p<0.05\right)$.

these results. A much dense, rigid structure and less swelling were expected by increasing of NVP from 20 to $40 \mathrm{wt} \%$ due to the less of DC\% (ESD: 450\%). Extra and unreacted NVP either remain among the network chains or in solution in PVP form; therefore, their sol fractions are more than the film with $20 \mathrm{wt} \%$ NVP. This concentration is chosen as an optimum concentration for cross-linking agent.

3.4. Water Vapor Transmission Rate. An optimal value of WVTR provides an ideal environment for wound bed; therefore, preparing a wound dressing with improved healing potential is an interesting subject [33]. The WVTR results for different formulations of the blend Ch/PEGF with 80/20 ratio are shown in Figure 4. Photocuring and increasing in the cross-linking agent concentration make networks denser; therefore, diffusion and transportation of water vapor decrease through the films. In other words, when a film was placed in a moisture rich environment the hydrated film facilitates vapor transfer from that environment to a dry environment. In these photocured blend films, the interactions of the permeating water molecules with the polar groups in the Ch film should not be discarded [1]. On the other hand, it could be due to the existence in intermolecular interactions between $\mathrm{Ch}$ and PEGF molecules [34]. The results, ranging from $553 \pm 53$ to $733 \pm 55 \mathrm{~g} \mathrm{~m}^{-2} \mathrm{day}^{-1}$, are comparable with the commercially available wound dressings in market such as Tegaderm and Op Site with $491 \pm 41$ and $792 \pm 32 \mathrm{~g} \mathrm{~m}^{-2}$ day $^{-1}$, respectively $[4,35]$. Considering accepted optimal WVTR value, these photocured films are promising candidates for dressing the wounds with low exudate.

3.5. Antibacterial Assay. It was reported that $\mathrm{Ch}$ is a bacteriostatic and bactericidal agent to 297 bacterial strains [36].

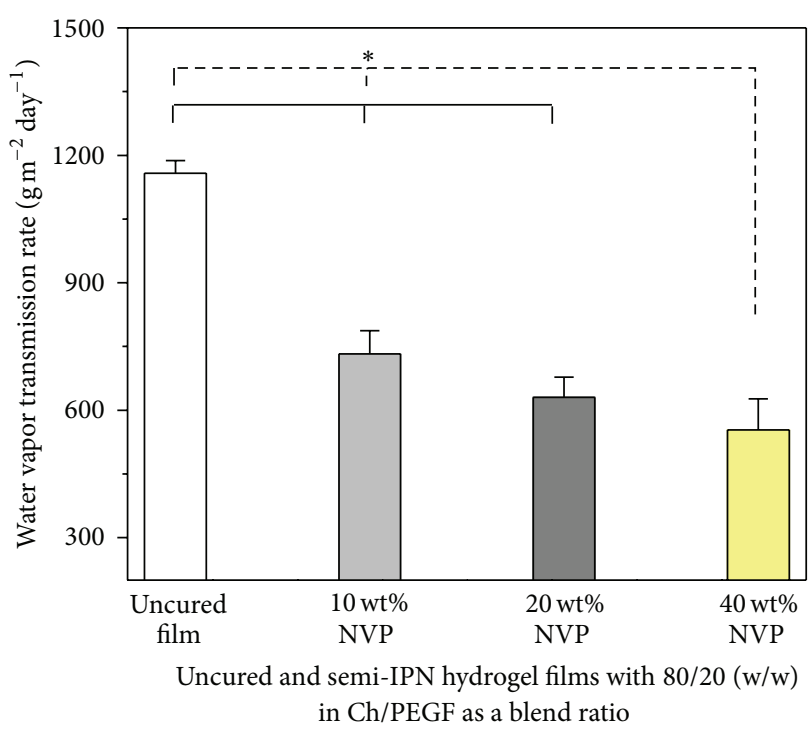

FIGURE 4: WVTR of photocured blend films of Ch/PEGF with 80/20 in blend ratio containing different contents of NVP and uncured Ch/PEGF with $80 / 20\left({ }^{*} p<0.05\right)$.

TABLE 1: Kill\% and population reduction against $S$. aureus values of semi-IPN hydrogels of Ch/PEGF and uncured blend $(n=3)$.

\begin{tabular}{lcc}
\hline $\begin{array}{l}\text { NVP (wt\%) in } \\
\text { Ch/PEGF }(80 / 20)\end{array}$ & $\begin{array}{c}\text { Kill\% } \\
\text { S. aureus }\end{array}$ & $\begin{array}{c}\text { Population reduction } \\
\left(\log _{10} \mathrm{CFU} \mathrm{mL}^{-1}\right)\end{array}$ \\
\hline 0 & $99.90 \pm 0.02^{*}$ & $3.02 \pm 0.11$ \\
10 & $99.86 \pm 0.02$ & $2.87 \pm 0.08$ \\
20 & $99.88 \pm 0.02$ & $2.83 \pm 0.09$ \\
40 & $99.81 \pm 0.01^{*}$ & $2.73 \pm 0.04$ \\
\hline
\end{tabular}

$\left({ }^{*} p<0.05\right)$.

Therefore, antibacterial assay was carried out for investigating whether semi-IPN hydrogels of Ch/PEGF exhibited antibacterial activity or not. The antibacterial activities as population reduction and killing efficacy (Kill\%) of S. aureus for uncured and cured films are summarized in Table 1. All test samples showed antibacterial activity against $S$. aureus by killing efficiency of more than $99 \%$ (99.81-99.86\%) and population reduction of bacterial load in the range of 2.73$2.87 \log \mathrm{CFU} \mathrm{mL} \mathrm{m}^{-1}$ (more than 1) [37]. The killing efficiency of the blend film with $40 \mathrm{wt} \%$ NVP showed significant difference compared with the uncured blend film. Some decrements were seen in antibacterial activity and population reduction; however, these differences were not statistically significant $(p>0.05)$. It may be due to the entrapment of surface amino groups of $\mathrm{Ch}$ chains in the semi-IPN hydrogel resulting in reduction of amino groups interactions with bacterial cell wall. In other words, the susceptibility of $\mathrm{Ch}$ active sites in the formulation of the film plays an important role in inhibiting the growth of this test organism. This claim is also applicable for miscible blends. In these blends, polymeric chains are homogenously distributed having more chances for binding with bacterial cells and killing them [10]. The real mechanism for antibacterial effect of these films 


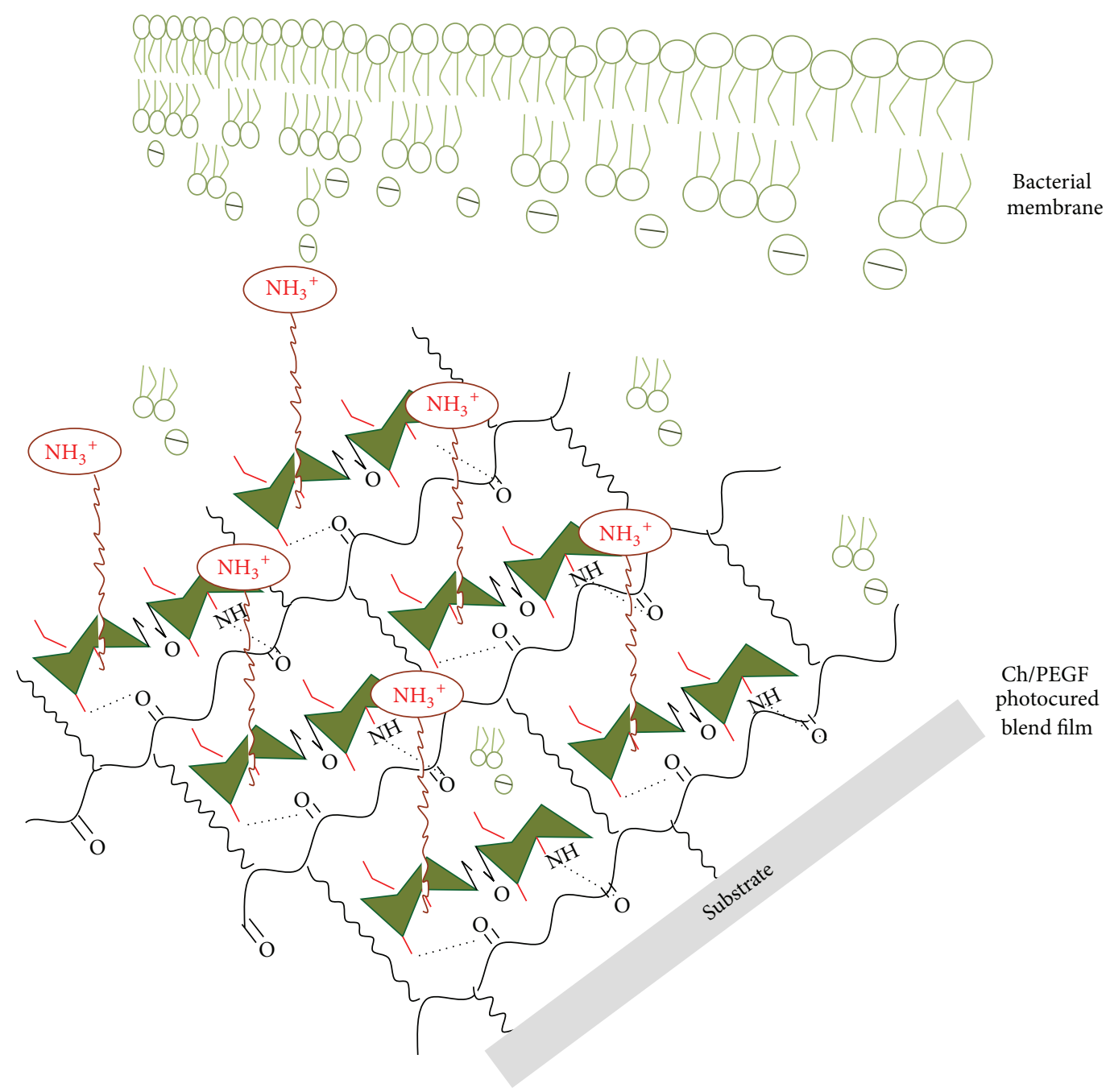

SCHEME 2: A suggested mechanism for antibacterial activity of photocured blend films of Ch/PEGF.

was not so clear [38] and needs more explorations; however, a suggested mechanism for cured films would be similar to uncured ones [1]. That is explained by interactions of cationic amino groups $\left(\mathrm{NH}_{3}{ }^{+}\right)$of Ch with negatively charged essential components of bacterial cell walls (Scheme 2). These interactions lead to destabilization of bacterial cell walls by disrupting of anionic phospholipid moieties of bacterial cell walls and consequent microbial death (Scheme 2). Li et al. reported the same mechanism for quaternized chitosan hydrogel films [27]. These claims need comprehensive tests to be confirmed which is beyond the scope of this study.

3.6. Cell Culture. Since an ideal wound dressing should be biocompatible as well as nontoxic, biocompatibility and cytocompatibility of hydrogel films were investigated. Optical images of fibroblast L929 cultured on the semi-IPN hydrogels of Ch/PEGF blends (80/20) with different concentrations of NVP after $24 \mathrm{~h}$ incubation at $37^{\circ} \mathrm{C}$ are shown in Figure 5. The images showed that cell attachment and proliferation were observed even in the presence of photocured films. Cross-linking makes 3D environment which could increase proper sites for cell anchoring, growth, and propagation. It has been also reported that good cell attachment does not happen in hydrophilic and hydrophobic surfaces [39] while PEGs with more than $2 \mathrm{kDa}$ are resistant to cell and proteins attachments [40]. It is expected that PEGF should be resistant to cell attachment. Once there is a balance between being hydrophilic and polycationic, cell attachment and propagation happened. Similar trend happens to uncured Ch/PEGF blends [1].

An appropriate wound dressing should not release toxic materials. Mitochondrial activity of fibroblast cells L929 on the film surface was evaluated by MTT assay. The cell viabilities of fibroblast L929 in contact with semi-IPN hydrogel films in 80/20 in blend ratio containing different concentrations of NVP after $24 \mathrm{~h}$ are shown in Figure 6. Results showed all films were nontoxic and kept their properties approximately similar to Ch films. According to Figure 6, 

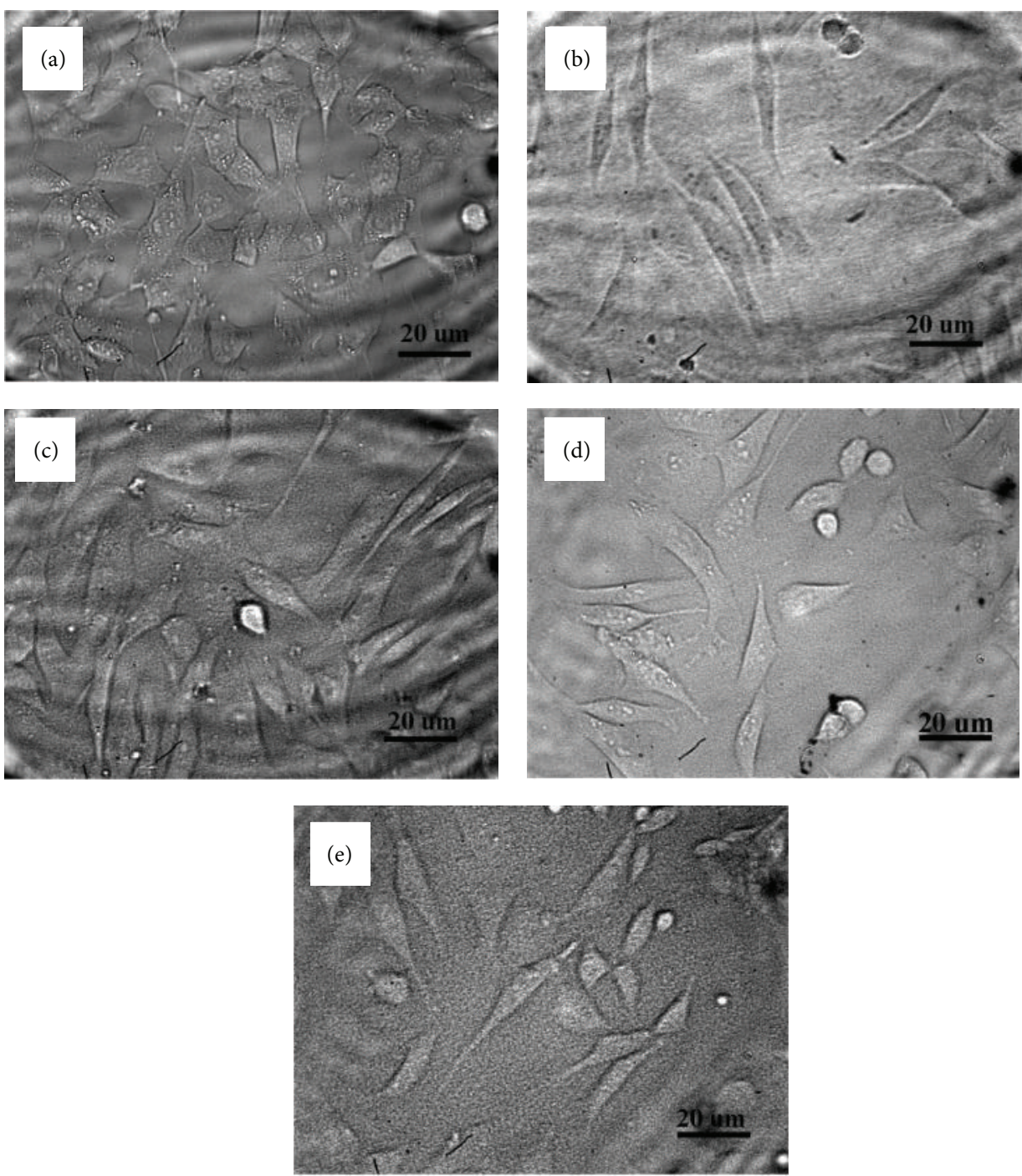

Figure 5: Optical microscopic images of fibroblast L929 on the control (a), uncured Ch/PEGF with 80/20 in blend ratio (b), and photocured films containing $10 \mathrm{wt} \%$ (c), $20 \mathrm{wt} \%$ (d), and $40 \mathrm{wt} \%$ (e) of NVP with magnification of 400x (scale: $20 \mu \mathrm{m}$ ) after $24 \mathrm{~h}$ of incubation.

the content of NVP in the blend film could influence the cell viability; the more the NVP concentration, the less the cell viability for which optical microscopic images confirmed MTT results. It may be due to the equilibrium water absorption and more susceptible nutrient media which are key factors needed for cell anchoring, growth, and immigration. Thus, less NVP concentration makes the network looser resulting in more water absorption.

\section{Conclusions}

In the present study the semi-IPN hydrogel films of Ch/PEGF were prepared using visible photocuring technique. FTIR was used to investigate the double bond conversion of PEGF section in Ch matrix for estimating the efficacy of the curing reaction in different cross-linking agent concentrations. The effect of NVP content on the biological and physicochemical properties of semi-IPN hydrogels of Ch/PEGF was evaluated. The increase in the NVP concentration from 10 to $40 \mathrm{wt} \%$ led to the decrease of antimicrobial activities against $S$. aureus but the difference was not statistically significant. MTT assay and cell culture indicated all samples were nontoxic and biocompatible to fibroblast L929. Not only did preparation of semi-IPN improve physicochemical properties but also biological properties were not destroyed. Finally, the antibacterial and biocompatible film cured with $20 \mathrm{wt} \% \mathrm{NVP}$ with the highest swelling degree and the lowest sol fraction was a suitable candidate as a hydrogel dressing for wounds with low exudates.

\section{Conflict of Interests}

The authors declare that there is no conflict of interests regarding the publication of this paper. 


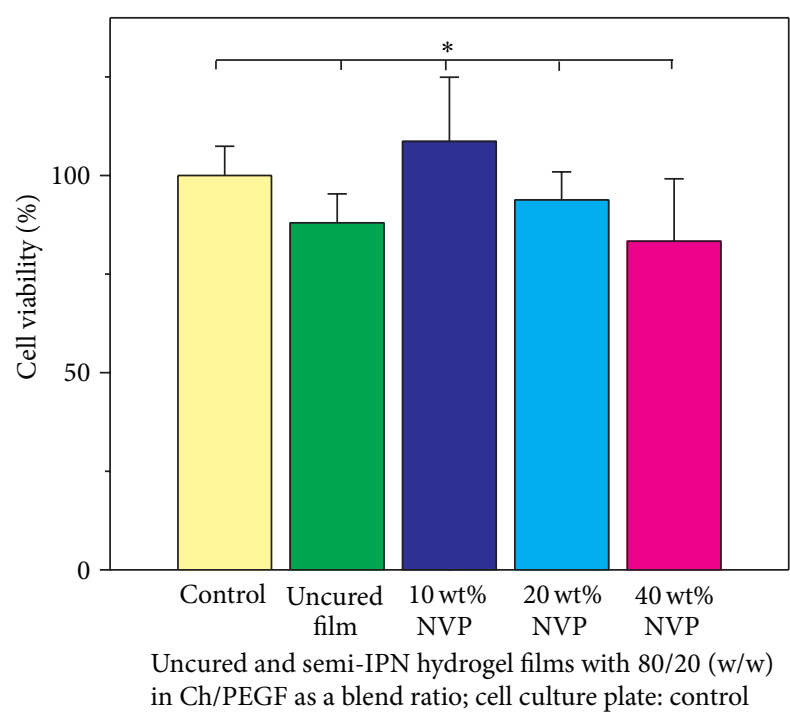

FIGURE 6: Cell viability of fibroblast L929 in contact with control and uncured Ch/PEGF film of 80/20 in blend ratio and photocured blend containing different contents of NVP after $24 \mathrm{~h}$ of incubation $(n=3)\left({ }^{*} p<0.05\right)$.

\section{Acknowledgments}

The authors would like to express their sincere gratitude to Iran National Science Foundation (no. 89001749). Also great thanks are due to Dr. M. Daliri Joupari (National Institute of Genetic Engineering and Biotechnology) and Mr. Hossein Jamalifar (Department of Drug and Food Control, Tehran University of Medical Sciences) for their kind help in cell and bacterial culture, respectively.

\section{References}

[1] A. H. Doulabi, H. Mirzadeh, M. Imani, and N. Samadi, "Chitosan/polyethylene glycol fumarate blend film: physical and antibacterial properties," Carbohydrate Polymers, vol. 92, no. 1, pp. 48-56, 2013.

[2] T. Wang, X.-K. Zhu, X.-T. Xue, and D.-Y. Wu, "Hydrogel sheets of chitosan, honey and gelatin as burn wound dressings," Carbohydrate Polymers, vol. 88, no. 1, pp. 75-83, 2012.

[3] J. S. Boateng, K. H. Matthews, H. N. E. Stevens, and G. M. Eccleston, "Wound healing dressings and drug delivery systems: a review," Journal of Pharmaceutical Sciences, vol. 97, no. 8, pp. 2892-2923, 2008.

[4] J. J. Elsner, A. Shefy-Peleg, and M. Zilberman, "Novel biodegradable composite wound dressings with controlled release of antibiotics: Microstructure, mechanical and physical properties," Journal of Biomedical Materials Research-Part B: Applied Biomaterials, vol. 93, no. 2, pp. 425-435, 2010.

[5] R. A. A. Muzzarelli, F. Greco, A. Busilacchi, V. Sollazzo, and A. Gigante, "Chitosan, hyaluronan and chondroitin sulfate in tissue engineering for cartilage regeneration: a review," Carbohydrate Polymers, vol. 89, no. 3, pp. 723-739, 2012.

[6] X. Ye, J. F. Kennedy, B. Li, and B. J. Xie, "Condensed state structure and biocompatibility of the konjac glucomannan/chitosan blend films," Carbohydrate Polymers, vol. 64, no. 4, pp. 532-538, 2006.
[7] Y. Wan, Y. Fang, H. Wu, and X. Cao, "Porous polylactide/chitosan scaffolds for tissue engineering," Journal of Biomedical Materials Research Part A, vol. 80, no. 4, pp. 776-789, 2007.

[8] Q. Wang, N. Zhang, X. Hu, J. Yang, and Y. Du, "Chitosan/polyethylene glycol blend fibers and their properties for drug controlled release," Journal of Biomedical Materials Research Part A, vol. 85, no. 4, pp. 881-887, 2008.

[9] C. Santos, C. J. Silva, Z. Büttel et al., "Preparation and characterization of polysaccharides/PVA blend nanofibrous membranes by electrospinning method," Carbohydrate Polymers, vol. 99, pp. 584-592, 2014.

[10] S. Zivanovic, J. Li, P. M. Davidson, and K. Kit, "Physical, mechanical, and antibacterial properties of chitosan/PEO blend films," Biomacromolecules, vol. 8, no. 5, pp. 1505-1510, 2007.

[11] J. M. Yang, W. Y. Su, T. L. Leu, and M. C. Yang, "Evaluation of chitosan/PVA blended hydrogel membranes," Journal of Membrane Science, vol. 236, no. 1-2, pp. 39-51, 2004.

[12] N. E. Suyatma, A. Copinet, L. Tighzert, and V. Coma, "Mechanical and barrier properties of biodegradable films made from chitosan and poly (lactic acid) blends," Journal of Polymers and the Environment, vol. 12, no. 1, pp. 1-6, 2004.

[13] M. Spasova, N. Manolova, D. Paneva, and I. Rashkov, "Preparation of chitosan-containing nanofibres by electrospinning of chitosan/poly(ethylene oxide) blend solutions," e-Polymers, vol. 4, no. 1, pp. 624-635, 2004.

[14] N. Manolova, I. Rashkov, and A. Domard, "Chitosan/polyoxyethylene diacid films for drug release," Journal of Bioactive and Compatible Polymers, vol. 12, no. 3, pp. 221-230, 1997.

[15] M. Ignatova, N. Manolova, N. Markova, and I. Rashkov, "Electrospun non-woven nanofibrous hybrid mats based on chitosan and PLA for wound-dressing applications," Macromolecular Bioscience, vol. 9, no. 1, pp. 102-111, 2009.

[16] A. Hashemi Doulabi, H. Mirzadeh, and M. Imani, "Interaction and miscibility study of fumarate-based macromers with chitosan," Materials Chemistry and Physics, vol. 139, no. 2-3, pp. 515-524, 2013.

[17] H. Shin, J. S. Temenoff, and A. G. Mikos, "In vitro cytotoxicity of unsaturated oligo[poly(ethylene glycol)fumarate] macromers and their cross-linked hydrogels," Biomacromolecules, vol. 4, no. 3, pp. 552-560, 2003.

[18] A. S. H. Doulabi, H. Mirzadeh, M. Imani, S. Sharifi, M. Atai, and S. Mehdipour-Ataei, "Synthesis and preparation of biodegradable and visible light crosslinkable unsaturated fumarate-based networks for biomedical applications," Polymers for Advanced Technologies, vol. 19, no. 9, pp. 1199-1208, 2008.

[19] P. Kolhe and R. M. Kannan, "Improvement in ductility of chitosan through blending and copolymerization with PEG: FTIR investigation of molecular interactions," Biomacromolecules, vol. 4, no. 1, pp. 173-180, 2003.

[20] M. Zhang, X. H. Li, Y. D. Gong, N. M. Zhao, and X. F. Zhang, "Properties and biocompatibility of chitosan films modified by blending with PEG," Biomaterials, vol. 23, no. 13, pp. 2641-2648, 2002.

[21] A. S. H. Doulabi, S. Sharifi, M. Imani, and H. Mirzadeh, "Synthesis and characterization of biodegradable in situ forming hydrogels via direct polycondensation of poly(ethylene glycol) and fumaric acid," Iranian Polymer Journal (English Edition), vol. 17, no. 2, pp. 125-133, 2008.

[22] A. Hashemi Doulabi, H. Mirzadeh, and M. Imani, "Miscibility study of chitosan/polyethylene glycol fumarate blends in dilute solutions," Journal of Applied Polymer Science, vol. 127, no. 5, pp. 3514-3521, 2013. 
[23] M. Atai, D. C. Watts, and Z. Atai, "Shrinkage strain-rates of dental resin-monomer and composite systems," Biomaterials, vol. 26, no. 24, pp. 5015-5020, 2005.

[24] ASTM, "Standard test methods for water vapor transmission of materials," ASTM Standards, ASTM International, West Conshohocken, Pa, USA, 2010.

[25] A. R. Sarasam, R. K. Krishnaswamy, and S. V. Madihally, "Blending chitosan with polycaprolactone: effects on physicochemical and antibacterial properties," Biomacromolecules, vol. 7, no. 4, pp. 1131-1138, 2006.

[26] J. M. Yang, H. T. Lin, T. H. Wu, and C.-C. Chen, "Wettability and antibacterial assessment of chitosan containing radiationinduced graft nonwoven fabric of polypropylene-g-acrylic acid," Journal of Applied Polymer Science, vol. 90, no. 5, pp. 1331-1336, 2003.

[27] P. Li, Y. F. Poon, W. Li et al., "A polycationic antimicrobial and biocompatible hydrogel with microbe membrane suctioning ability," Nature Materials, vol. 10, no. 2, pp. 149-156, 2011.

[28] M. Farhadi, H. Mirzadeh, A. Solouk et al., "Collagenimmobilized patch for repairing small tympanic membrane perforations: in vitro and in vivo assays," Journal of Biomedical Materials Research Part A, vol. 100, no. 3, pp. 549-553, 2012.

[29] S. Sharifi, Y. Shafieyan, H. Mirzadeh et al., "Hydroxyapatite scaffolds infiltrated with thermally crosslinked polycaprolactone fumarate and polycaprolactone itaconate," Journal of Biomedical Materials Research-Part A, vol. 98, no. 2, pp. 257-267, 2011.

[30] N. Emami and K.-J. M. Söderholm, "Influence of light-curing procedures and photo-initiator/co-initiator composition on the degree of conversion of light-curing resins," Journal of Materials Science: Materials in Medicine, vol. 16, no. 1, pp. 47-52, 2005.

[31] S. Sharifi, H. Mirzadeh, M. Imani et al., "Synthesis, photocrosslinking characteristics, and biocompatibility evaluation of $\mathrm{N}$-vinyl pyrrolidone/polycaprolactone fumarate biomaterials using a new proton scavenger," Polymers for Advanced Technologies, vol. 19, no. 12, pp. 1828-1838, 2008.

[32] S. Jo, H. Shin, A. K. Shung, J. P. Fisher, and A. G. Mikos, "Synthesis and characterization of oligo(poly(ethylene glycol) fumarate) macromer," Macromolecules, vol. 34, no. 9, pp. 28392844, 2001.

[33] B. Balakrishnan, M. Mohanty, P. R. Umashankar, and A. Jayakrishnan, "Evaluation of an in situ forming hydrogel wound dressing based on oxidized alginate and gelatin," Biomaterials, vol. 26, no. 32, pp. 6335-6342, 2005.

[34] N. Gontard and S. Guilbert, Food Packaging and Preservation, Blackie Academic \& Professional, London, UK, 1st edition, 1994.

[35] H.-J. Yoo and H.-D. Kim, "Characteristics of waterborne polyurethane/poly( $N$-vinylpyrrolidone) composite films for wound-healing dressings," Journal of Applied Polymer Science, vol. 107, no. 1, pp. 331-338, 2008.

[36] R. A. A. Muzzarelli, R. Tarsi, O. Filippini, E. Giovanetti, G. Biagini, and P. E. Varaldo, "Antimicrobial properties of Ncarboxybutyl chitosan," Antimicrobial Agents and Chemotherapy, vol. 34, no. 10, pp. 2019-2023, 1990.

[37] R. J. B. Pinto, S. C. M. Fernandes, C. S. R. Freire et al., "Antibacterial activity of optically transparent nanocomposite films based on chitosan or its derivatives and silver nanoparticles," Carbohydrate Research, vol. 348, pp. 77-83, 2012.

[38] Y. Feng and W. Xia, "Preparation, characterization and antibacterial activity of water-soluble O-fumaryl-chitosan," Carbohydrate Polymers, vol. 83, no. 3, pp. 1169-1173, 2011.
[39] C. J. Wilson, R. E. Clegg, D. I. Leavesley, and M. J. Pearcy, "Mediation of biomaterial-cell interactions by adsorbed proteins: a review," Tissue Engineering, vol. 11, no. 1-2, pp. 1-18, 2005.

[40] B. D. Ratner, A. S. Hoffman, F. J. Schoen, and J. E. Lemons, Biomaterials Science. An Introduction to Materials in Medicine, Elsevier Academic Press, San Diego, Calif, USA, 2004. 

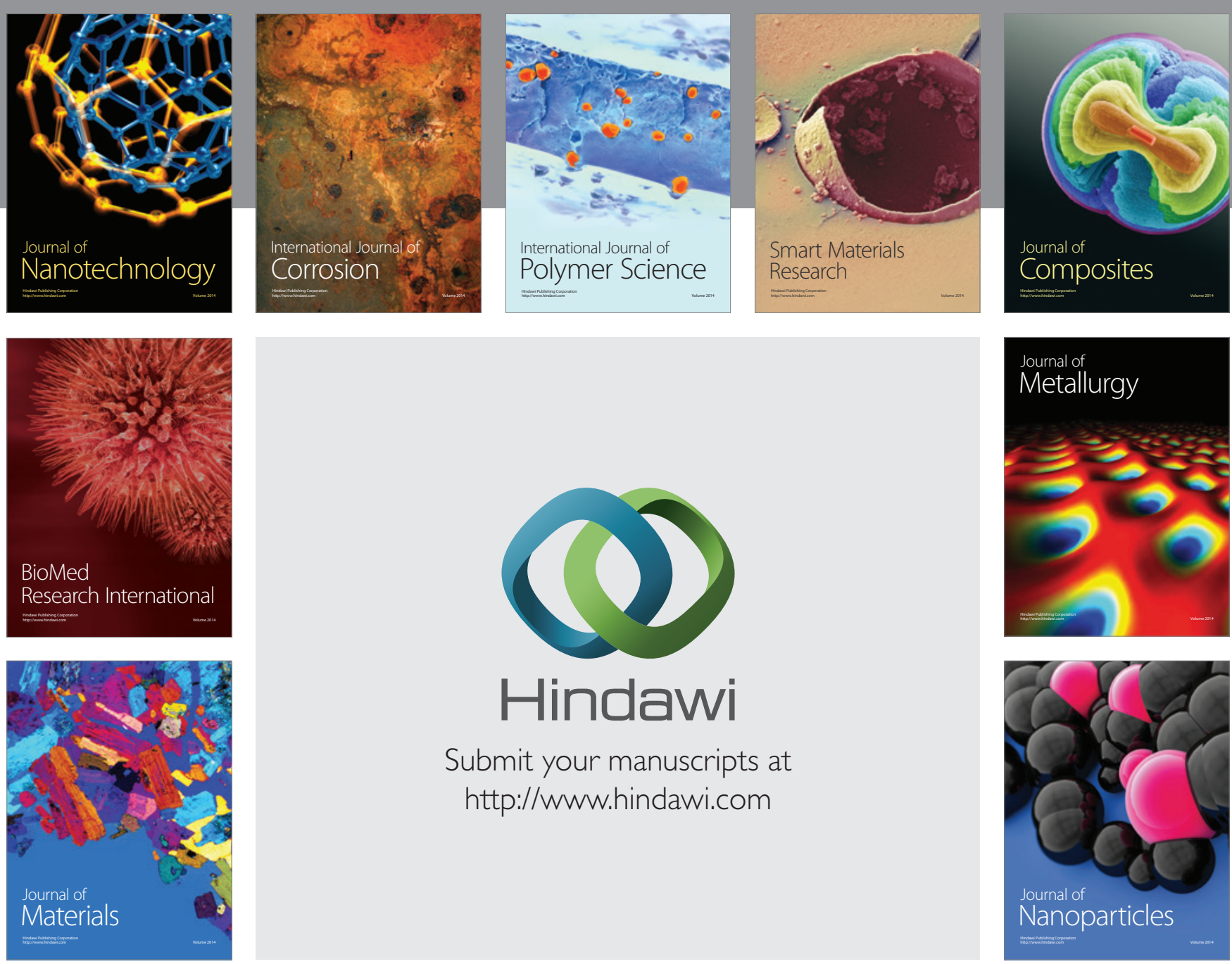

Submit your manuscripts at http://www.hindawi.com
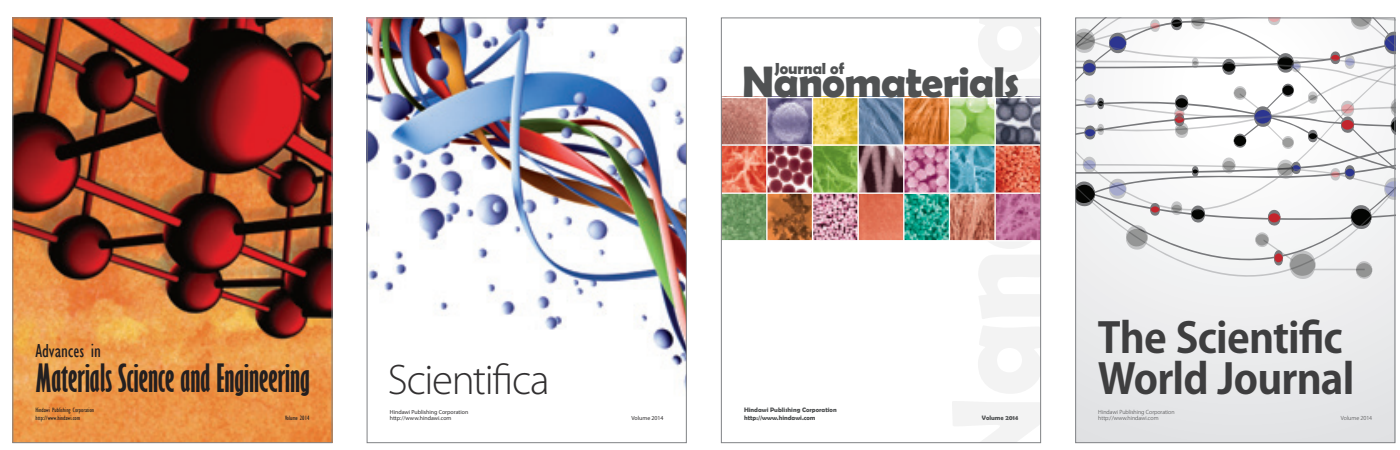

\section{The Scientific World Journal}
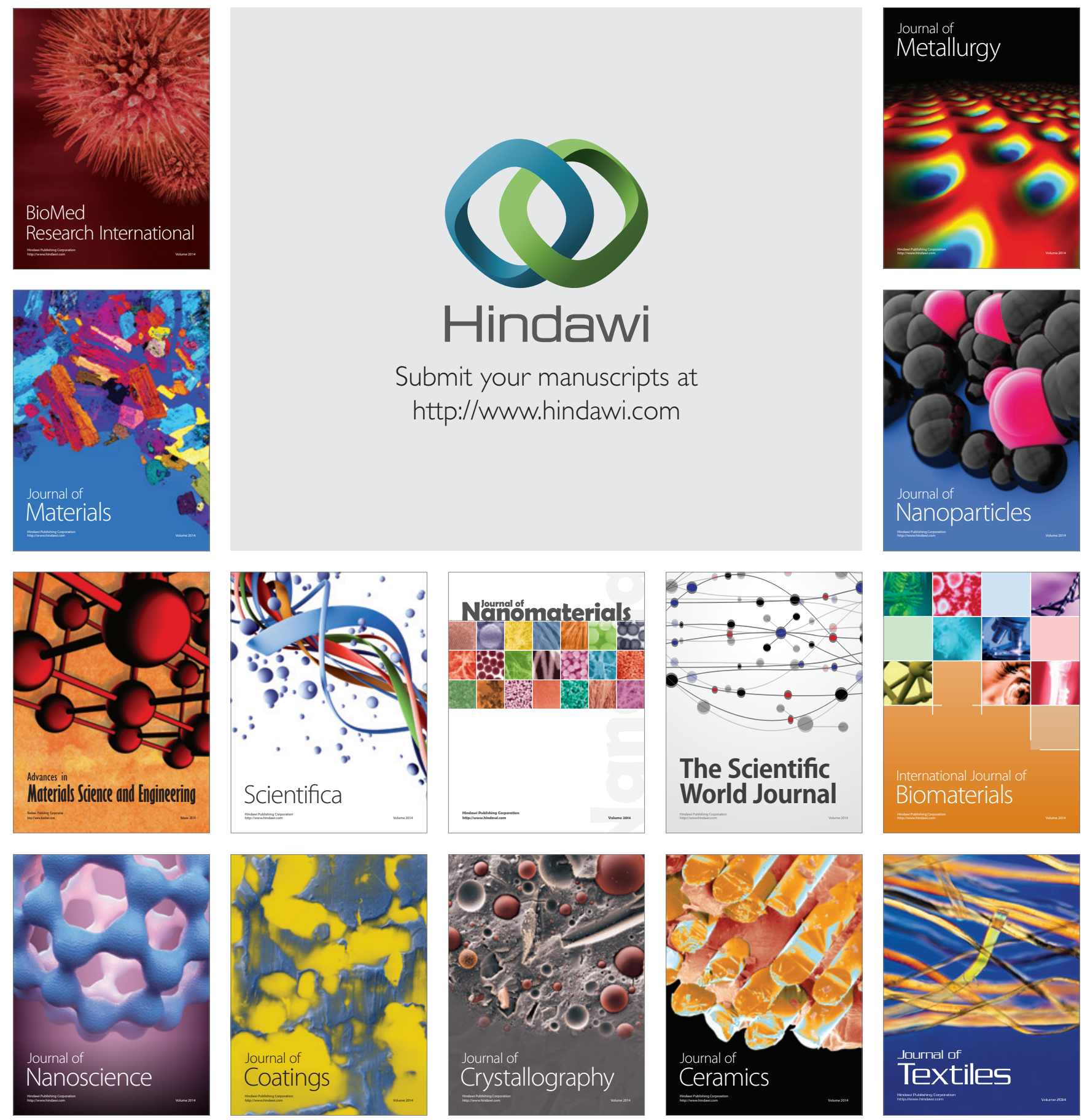